\title{
Photoluminescence study of terbium-exchanged ultrastable Y zeolites: Number of species, photoluminescence decays, and decay-associated spectra
}

\author{
Carmen Tiseanu, ${ }^{1, a)}$ V. A. Lórenz-Fonfría, ${ }^{2, b)}$ V. I. Parvulescu, ${ }^{3}$ A. Gessner, ${ }^{4}$ and \\ M. U. Kumke ${ }^{4}$ \\ ${ }^{1}$ National Institute for Laser, Plasma and Radiation Physics, P.O. Box MG-36, RO 76900, Bucharest- \\ Magurele, Romania \\ ${ }^{2}$ Department of Materials Science and Engineering, Nagoya Institute of Technology, Showa-ku, 466-855 \\ Nagoya, Japan \\ ${ }^{3}$ Department of Chemical Technology and Catalysis, University of Bucharest, 4-12 Regina Elisabeta Bvd., \\ Bucharest 030016, Romania \\ ${ }^{4}$ Institute of Chemistry, Physical Chemistry, University of Potsdam, Karl-Liebknecht-Str. 24-25, 14476 \\ Potsdam-Golm, Germany
}

(Received 24 November 2007; accepted 9 June 2008; published online 12 August 2008)

\begin{abstract}
Terbium-exchanged ultrastable Y (USY) zeolites were investigated by using time-resolved photoluminescence spectroscopy techniques and methods. To determine the distribution of terbium species in USY zeolites together with their photoluminescence properties, several analysis methods for the time-resolved luminescence spectra were used such as the area normalization of time-resolved photoluminescence spectra, singular value decomposition, global nonlinear least squares, and the maximum entropy. Except for a questionable long lifetime, small contribution of a terbium species with lifetime of 1.9-2.1 ms, all the experimental and analysis results converged to a two terbium species distribution with lifetimes varying between 410-440 and 1000-1100 $\mu$ s. The effects of the silylation of terbium-exchanged USY zeolites with phenyl-, vinyl-, and hexadecyltrimethoxysilanes on the lanthanide's photoluminescence properties were also described. (C) 2008 American Institute of Physics. [DOI: 10.1063/1.2966304]
\end{abstract}

\section{INTRODUCTION}

Lanthanides exchanged sol-gel derived materials such as zeolites are intensively investigated due to their potential photoluminescence (PL)-based applications. ${ }^{1-4}$ Besides high quantum PL efficiency, other key parameters considered in the preparation of luminescent zeolite-type materials are the distribution of the lanthanide's species and the nature of the local microenvironment at the dopants sites. PL spectroscopy has proved to be a sensitive method for the description of the local environment surrounding the lanthanide ions in zeolites in terms of the PL spectra and decays. Generally, a nonexponential shape of the PL decays of the lanthanides (typically europium or terbium ions) is obtained in line with the heterogeneous nature of these materials. However, to convene the mathematical data fitting of the PL decay curves with a physical model is not straightforward. The PL dynamics of the lanthanide's exchanged sol-gel derived materials can be modeled as a multiexponential decay (generally by fitting with two or three discrete exponential decay lifetimes) or as a continuous distribution of the PL decay times. The energy transfer to the various defects in the matrix or concentration quenching mechanisms can further complicate the decay shape at short times. We have recently underlined the need for caution when interpreting nonexponential decays of lan-

\footnotetext{
a) Author to whom correspondence should be addressed. Electronic mail:tiseanuc@yahoo.com.

${ }^{b)}$ On leave from Department of Materials and Science Engineering, Nagoya Institute of Technology, Showa-ku, 466-855 Nagoya, Japan.
}

thanides in microporous/mesoporous materials in terms of a discrete distribution of the emissive species. ${ }^{5-7}$ A combined information from both the spectral and the time domain as that provided by time-resolved PL spectra can bring more reliable information concerning distribution of lanthanides in these heterogeneous materials. It is acknowledged that the quantum PL efficiency obtained with lanthanides in zeolites is strongly affected by the nonradiative relaxation via the vibrational coupling between the electronic levels of the lanthanide ion with the - $\mathrm{OH}$ groups inherently present in a solgel matrix. ${ }^{8}$ Albeit the PL properties can be improved following a thermal treatment at high temperatures or calcination they are subject to full or partial restoration to those measured in the hydrated state upon exposure to the moist atmosphere. ${ }^{5,6}$ To decrease the adverse effects of water on the PL properties of the lanthanides-exchanged zeolites, the surface of zeolites can be made hydrophobic by the silylation technique. ${ }^{7,9-11}$

Here we present a detailed time-resolved PL study of nonsilylated and silylated terbium exchanged into dealuminated or ultrastable Y (USY) zeolites. Silylation was used as method to retard the intrusion of water or atmosphere moist at the terbium sites and hence to preserve terbium PL properties close to the values obtained after calcination. The aim of the paper is to identify the number and PL properties of the terbium species in the non-silylated and silylated USY zeolites. To determine the number of species present we used singular value decomposition ${ }^{12}$ (SVD) as well as area normalization of time-resolved emission spectra (TRANES). ${ }^{6,13}$ 


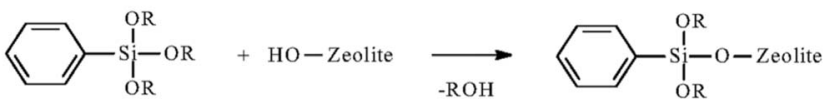

SCHEME 1. Postgrafting of the organic groups onto zeolite surface.

To determine and characterize the number, time constants, and amplitude spectra decay from the exponential data, we used both exponential fitting by global nonlinear least-squares ${ }^{14-17}$ and lifetime distribution recovery by the maximum entropy and Monte Carlo method. ${ }^{18-22}$ From the comparison between the terbium PL properties in the nonsilylated and silylated USY zeolites, the effects of the silylation were also inferred.

\section{EXPERIMENTAL}

\section{A. Samples synthesis and characterization}

$\mathrm{Tb}^{3+}$-exchanged zeolites (CBV-720, USY, Si $/ \mathrm{Al}=15$, from PQ Zeolites Corporation) were prepared following a typical ion-exchange procedure. ${ }^{23}$ In accordance, $1 \mathrm{~g}$ of USY was added in $25 \mathrm{ml}$ of $0.004 \mathrm{M} \mathrm{Tb}^{3+}$ aqueous solution and stirred for $30 \mathrm{~min}$ at $80{ }^{\circ} \mathrm{C}$. The samples were then recuperated, washed until free from terbium ions, and dried in air at $50{ }^{\circ} \mathrm{C}$ for 2 days. Before analysis, the samples have been treated following two different routes: (i) calcination of the ion-exchanged zeolites at $400{ }^{\circ} \mathrm{C}$ for $4 \mathrm{~h}$ and (ii) the hydrophobization of the external surface of the calcined $\mathrm{Tb}^{3+}$-exchanged zeolites with phenyl-, vinyl-, and hexadecyltrimethoxysilanes. A conventional grafting technique was applied (see Scheme 1).

Briefly, a suspension of $1 \mathrm{~g}$ calcined terbium zeolite was dispersed in $100 \mathrm{ml}$ of toluene (previously dried with $5^{a}$ molecular sieves) under vigorous stirring. Next, $20 \mathrm{ml}$ of trimethoxysilane derivatives (used as received) were added to this solution under continuous stirring at room temperature for $24 \mathrm{~h}$. The grafted zeolites were then filtered, washed with toluene, and then dried at $100{ }^{\circ} \mathrm{C}$ under vacuum. The materials thus obtained were denoted as USY $/ \mathrm{Tb}^{3+}$ (the parent zeolite) and USY $/ \mathrm{Tb}^{3+} /$ vinyl, USY $/ \mathrm{Tb}^{3+} /$ phenyl and $\mathrm{USY} / \mathrm{Tb}^{3+} / \mathrm{C}_{16}$, for the silylated zeolites. Structural characterization of the investigated samples was performed using nitrogen adsorption isotherms at $-196{ }^{\circ} \mathrm{C}$, thermogravimetric analysis, Fourier transform infrared (FT-IR) spectroscopy, diffuse reflectrance infrared Fourier-transform spectroscopy (DRIFTS), x-ray diffraction (XRD), and DR-UV-VIS spectroscopy. The specific surface areas of the samples and micropore volume were determined using the micropore program from nitrogen adsorption isotherms collected at $-196{ }^{\circ} \mathrm{C}$ with a Micromeritics ASAP2020 instrument, after degassing the samples in situ at $120{ }^{\circ} \mathrm{C}$ for $5 \mathrm{~h}$. Powder XRD (PXRD) patterns were collected on a Siemens D-5000, using $\mathrm{Cu} K \alpha$ radiation $(\lambda=1.54050 \AA)$ and quartz as an external standard. FT-IR spectra were collected in a Nicolet 4700 FT-IR instrument using the $\mathrm{KBr}$ pellet technique; 100 scans were averaged to improve the signal-to-noise ratio, at a nominal resolution of $4 \mathrm{~cm}^{-1}$. DRIFT spectra were collected with the same apparatus and the same resolution during the heating the samples in helium with a rate of $1{ }^{\circ} \mathrm{C} / \mathrm{min}$ with a plateau of $1 \mathrm{~h}$ at each measuring temperature. Thermogravimetric analysis curves (thermogravimetric and heat flow) were collected using a SETARAM 9216.18 apparatus in air using a heating rate of $10{ }^{\circ} \mathrm{C} / \mathrm{min}$. DR-UV-visible spectra were collected with a Specord250 (AnalytikJena).

\section{B. Photoluminescence measurements}

TRES were recorded using a wavelength tunable Nd:YAG (yttrium aluminum garnet) laser/OPO system (Spectra Physics/Gwu) operating at $20 \mathrm{~Hz}$ as excitation light source and an intensified charge-coupled device camera (Andor Technology) coupled to a spectrograph (MS257 Model $77700 \mathrm{~A}$, Oriel Instruments) as detector. The detector provides a maximal spectral resolution of $0.08 \mathrm{~nm}$. For the TRES measurements, the box car technique was used, in which the PL signal is collected in a certain time interval (gate width) delayed after the laser flash (gate delay) with the gate delay nonlinearly increased in order to obtain closely spaced points at short times and more spaced points at longer time scales. In a typical experiment, the sample was excited at 337,390 , or $487 \mathrm{~nm}$, the initial gate delay was $5 \mu$ s, and the gate width $100 \mu \mathrm{s}$. The PL was detected from 400 to 700 $\mathrm{nm}$. Similar experimental conditions were used in the measurements for all terbium-USY zeolites. Following the calcination or silylation, all samples (parent or silylated) were allowed to rest under moist atmosphere. Reproducibility of the experimental data was found to be satisfactory as judged by the results of up to four experiments per sample. Unless specified, the presented results correspond to an excitation wavelength of $487 \mathrm{~nm}$.

\section{Photoluminescence data analysis}

Both PL spectra and decays were in-depth analyzed. To estimate the number of the present independent specie, timeresolved PL spectra were analyzed by two independent methods, e.g., area normalized TRES (TRANES) (Ref. 6 and 13) and SVD. The TRANES were constructed by normalizing the area of each spectrum in the 535-560 nm interval, such that the area of the spectrum at time $t$ is equal to the area of the spectrum at $t=5 \mu \mathrm{s}$. SVD decomposed TRES into orthonormal abstract spectra and abstract time traces, ordered by their singular values. ${ }^{12}$ For the error-free data, the singular values decay faster than exponentially, and the number of nonzero singular values gives the number of independent spectroscopic/kinetic components. In contrast, when the data contains only random Gaussian noise, the singular values decay exponentially. When the data contains both signal and random Gaussian noise, to obtain the number of signal singular value above the noise singular values, and thus estimate the number of independent spectroscopic/kinetic components within the noise is straightforward. SVD was performed in MATLAB, using the built in function "svd," and taking into account the observed noise standard deviation dependence on time. Unless otherwise stated, previous to any analysis, TRES were quasilogarithmically averaged to a maximum of twenty spectra per decade (peak normalized) or four spectra per decade (area normalized or TRANES). 
TABLE I. Physical adsorption data, mass loss and the content in the silylated organic ligand for the parent and silylated USY zeolites

\begin{tabular}{lcccc}
\hline \hline \multicolumn{1}{c}{ Zeolite } & $\begin{array}{c}\text { Langmuir } \\
\text { surface area }\left(\mathrm{m}^{2} / \mathrm{g}\right)\end{array}$ & $\begin{array}{c}\text { Micropore volume } \\
(\mathrm{ml} / \mathrm{g})\end{array}$ & $\begin{array}{c}\text { Mass loss } \\
\mathrm{g} / \mathrm{g} \text { zeolite }\end{array}$ & $\begin{array}{c}\text { Silylating agent loading, } \\
\text { mmoles/g zeolite }\end{array}$ \\
\hline $\mathrm{USY}$ & 498 & 0.39 & & \\
$\mathrm{USY} / \mathrm{Tb}^{3+}$ & 482 & 0.37 & & \\
$\mathrm{USY} / \mathrm{Tb}^{3+} /$ vinyl & 355 & 0.29 & 0.14 & 2.3 \\
$\mathrm{USY} / \mathrm{Tb}^{3+} / \mathrm{phenyl}$ & 378 & 0.26 & 0.18 & 1.8 \\
$\mathrm{USY} / \mathrm{Tb}^{3+} / \mathrm{C}_{16}$ & 315 & 0.18 & 0.26 & 1.3 \\
\hline \hline
\end{tabular}

The PL decays were analyzed by using three methods. (1) Fitting of the integrated PL decays within 535-560 nm wavelength interval with a multiexponential function, $f(t)$, as

$$
f(t)=\sum_{i=1}^{n} A_{i} \exp \left(-t / \tau_{i}\right)+B,
$$

where $A_{i}$ is the decay amplitude, $B$ is a constant (the baseline offset), and $\tau_{i}$ is the decay time. The average decay times were calculated using the following formula:

$$
\langle\tau\rangle=\sum_{i=1}^{n} A_{i} \tau_{i} / \sum_{i=1}^{n} A_{i} .
$$

(2) Global fitting to a multiexponential decay by using nonlinear least-squares (GExpNLLS). ${ }^{14-17}$ The PL decays at all emission wavenumbers within $535-560 \mathrm{~nm}$ were used. The method provides besides the exponential time constants of decay, the decay amplitude spectra (DAS). The DAS are the exponential amplitudes versus the wavelength for a given time constant of decay. ${ }^{16,17}$ In case of parallel PL decays, the amplitude spectra corresponds directly to pure PL spectra of the present species, scaled by their relative initial population. The time traces were weighted taking into account the estimated noise standard deviation dependence on time, whereas the standard dependency on wavelength was ignored. The data within range of 5-100 $\mu$ s were discarded in the analysis. GExpNLLS was performed following the LevenbergMarquard method ${ }^{24}$ in a program implemented in MATLAB v7. $^{20}$ (3) The maximum entropy method (MaxEnt). The TRES total area between 535 and $560 \mathrm{~nm}$ was analyzed by providing the distribution of exponential amplitudes versus exponential time constants. ${ }^{18,19}$ The number of bands recovered in the MaxEnt lifetime distribution represents the number of resolvable decay components in the data, whereas the band positions and band areas of the MaxEnt lifetime distribution give the corresponding time constants and amplitudes of the decaying components. The agreement between experimental and calculated data was measured using the $\chi^{2}$, with a noise standard deviation iteratively improved by re-using the residuals of a previous MaxEnt analysis. The generalized Shannon-Jaynes entropy for solution without sign restrictions was used. ${ }^{21}$ For an automatic and optimum selection of the regularization value, we used both the $L$-curve method and Bayesian inference with automatic noise scaling. ${ }^{20-22}$ In most cases, both methods recommended similar regularization values; otherwise, the optimum regularization value was manually selected between the limits provided by the meth- ods. Once a maximum entropy lifetime distribution was obtained, using of Monte Carlo simulations identified the noiseinduced features. Lifetime distribution analysis by MaxEnt, including the methods for automatic selection of the regularization value and the estimation of noise-induced errors in the lifetime distributions were implemented in MATLAB v7. ${ }^{20,21}$ From the obtained number and time constants of the decays, the DAS were obtained from the experimental data as a linear least-square step taking into account the estimated noise standard deviation dependence on time.

\section{RESULTS AND DISCUSSION}

\section{A. Structural characterization}

Due to the susceptibility of the Al-O bonds to hydrolysis, one of the issues associated with the use of zeolites is their stability in acidic and basic media and under hydrothermal conditions. Under such conditions, an extensive and uncontrolled removal of aluminum atoms from the zeolite lattice takes place, leading to a progressive collapse of the crystal structure. Following a controlled dealumination the proton form of faujasite (zeolite $\mathrm{Y}$ ) is converted to a very stable material, the so-called USY zeolite. Following silylation, the thermally treated or calcined USY zeolites showed different weight loss profiles compared to those of parent zeolites. As for the case of terbium-BETA zeolites, below $150{ }^{\circ} \mathrm{C}$ the parent terbium-USY zeolites loss their adsorbed water. ${ }^{7}$ Upon silylation, the adsorbed water decreased, with a minimum loss measured for the $\mathrm{C}_{16}$ silylated ones. The additional weight loss occurring until $500{ }^{\circ} \mathrm{C}$ is associated with the complete combustion of the organic molecules. Table I compiles the mass loss, the content in the silylated compound, and the textural characteristics of the investigated zeolites. Deposition of terbium caused a certain decrease in both surface area and micropore volume, due to a partial blockage of micropores. Further, silylation of $\mathrm{USY} / \mathrm{Tb}^{3+}$ with different trimethoxysilane derivatives led to an additional decrease of the surface area and micropore volume, which actually paralleled the size of the organic chain. A dramatic decrease of the surface area and micropore volume was determined when used a $\mathrm{C}_{16}$ radical, which corresponds to an advanced blockage of the internal surface of USY zeolite. Neither the ionic exchange or silylation caused any change in the crystallinity of these zeolites. Diffraction lines associated with parent zeolites have similar intensity in the silylated zeolites, irrespective of the organic molecule (figure not shown). The terbium content was too small to generate 

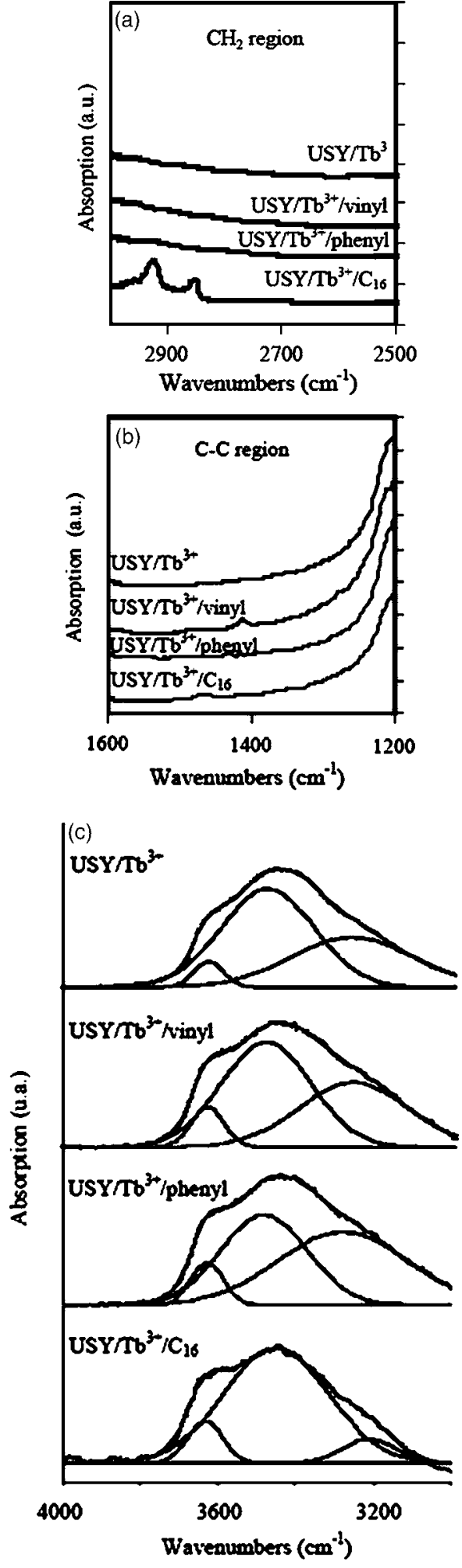

FIG. 1. FTIR spectra in the region of $\mathrm{C}-\mathrm{C}$ (a) and $\mathrm{CH}_{2}$ (b) bonds and (c) silanol region for $\mathrm{USY} / \mathrm{Tb}^{3+}$ zeolites.

visible XRD $\mathrm{Tb}_{2} \mathrm{O}_{3}$ particles. IR bands between 800 and $400 \mathrm{~cm}^{-1}$, associated with the investigated zeolites were detected in all samples confirming thus the results obtained with XRD. In addition, the silylated zeolites, presented the specific bands due to $\mathrm{C}-\mathrm{C}$ or $\mathrm{C}-\mathrm{H}$ bonds. Bands assigned to $\mathrm{CH}_{2}$ and $\mathrm{C}-\mathrm{C}$ bonds were identified as well [Figs. 1(a) and 1(b)]. Although in the way has been applied, the FT-IR tech-

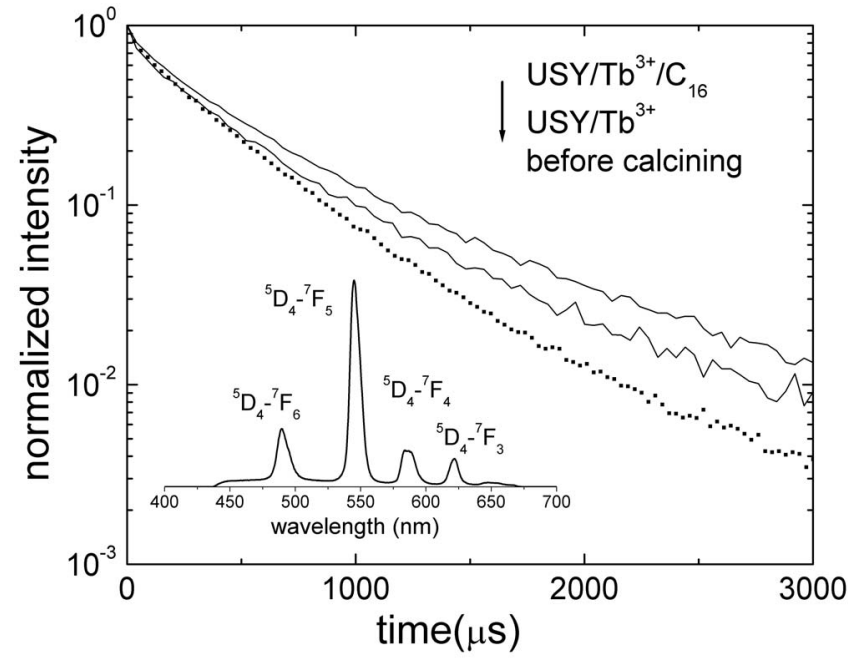

FIG. 2. PL spectrum of USY $/ \mathrm{Tb}^{3+} /$ vinyl zeolite following excitation at 487 nm. Comparison between the PL decays of parent and silylated (USY/ $\mathrm{Tb}^{3+} / \mathrm{C}_{16}$ ) zeolites. With dotted line is represented the PL decay of the as-exchanged (hydrated) $\mathrm{USY} / \mathrm{Tb}^{3+}$. Insert: $\mathrm{PL}$ spectrum of $\mathrm{USY} / \mathrm{Tb}^{3+} / \mathrm{vinyl}$ zeolite. Excitation wavelengths was $487 \mathrm{~nm}$. PL spectrum of USY $/ \mathrm{Tb}^{3+} /$ vinyl zeolite following excitation of $487 \mathrm{~nm}$.

nique is not suited for the quantitative analysis, the marked differences between bands intensity point to a high content in the organic part for the zeolites silylated with hexadecyltrimethoxysilane [Fig. 1(a)]. Further differences were detected in the silanol region $\left(3700-3400 \mathrm{~cm}^{-1}\right)$ where the $\mathrm{Tb}^{3+}$-exchanged zeolites presented the highest content in $\mathrm{Si}-\mathrm{OH}$ groups while the silylated ones have a lower content, especially for zeolites silylated with hexadecyltrimethoxysilanes [Fig. 1(c)]. The deconvolution of the large band located in the region 3700-3100 $\mathrm{cm}^{-1}$ accounts for the presence of three different $\mathrm{OH}$ groups, which according to the literature can be assigned to terminal silanol groups (band at $3640 \mathrm{~cm}^{-1}$ ), bridging $\mathrm{OH}$ groups with Brønsted acidity (band at $3450 \mathrm{~cm}^{-1}$ ), and $\mathrm{OH}$ groups attached to trivalent cations compensating the $\mathrm{Al}^{3+}$ charge (band at $3250 \mathrm{~cm}^{-1}$ ). ${ }^{25}$ The location of these bands at such low wavenumbers is due to the fact that the measurements were carried out in $\mathrm{KBr}$. No absorption bands assigned to $4 f-4 f$ transitions were detected in our DR-UV-visible spectra. However, these spectra show weak shoulders one located around 246-250 nm, which might be assigned to the presence of $\mathrm{OH}$ ions in the coordination sphere of terbium. ${ }^{26}$ The band located at $266 \mathrm{~nm}$ was detected only for silylated zeolites with agents containing phenyl groups, and is assigned to these species. The absence of any absorption edge at $400-600 \mathrm{~nm}$ represents a good confirmation of the absence of $\mathrm{Tb}^{4+}$ species and that all terbium exists as isolated $\mathrm{Tb}^{3+}$ species. ${ }^{26}$

\section{B. General photoluminescence properties}

A representative time-resolved PL spectrum of the silylated $\mathrm{Tb}^{3+} / \mathrm{USY}$ zeolites is illustrated in Fig. 2. The most intense PL band corresponding to the ${ }^{5} D_{4^{-}}{ }^{7} F_{5}$ transition (545 $\mathrm{nm})$ was used in the PL decay measurements. Three other peaks were observed at $491 \mathrm{~nm}\left({ }^{5} D_{4} \rightarrow{ }^{7} F_{6}\right), 586 \mathrm{~nm}\left({ }^{5} D_{4}\right.$ $\left.\rightarrow{ }^{7} F_{4}\right)$, and $624 \mathrm{~nm}\left({ }^{5} D_{4} \rightarrow{ }^{7} F_{3}\right)$. Except for the hexadecyltrimethoxysilane $\left(\mathrm{C}_{16}\right)$, we have found that the effects of 

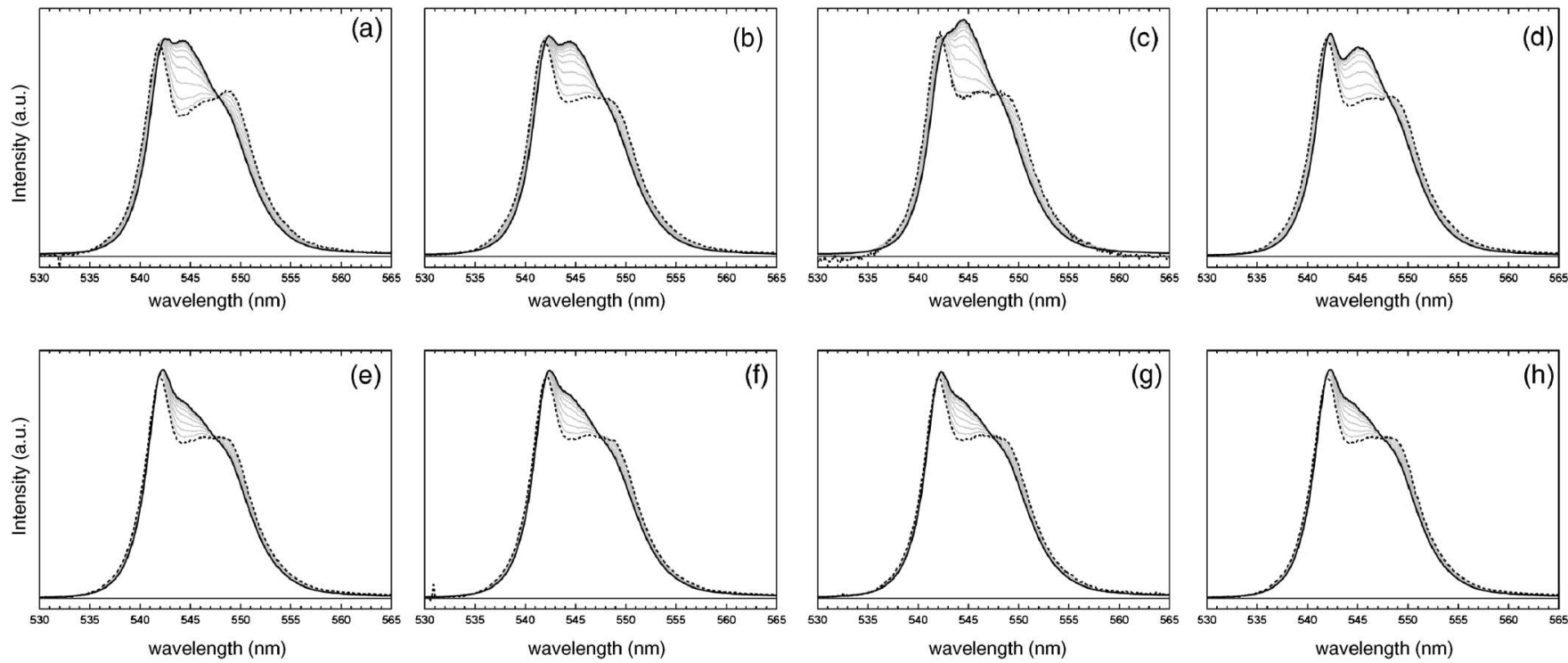

FIG. 3. TRANES at some selected times after the laser pulse: $5 \mu$ s (black continuous line), quasilogarithmically spaced between $8 \mu$ s and 3.7 ms (gray continuous lines), and at $6.7 \mathrm{~ms}$ (black dashed line). Excitation wavelengths: [(A(-)D)] $337 \mathrm{~nm}[(\mathrm{E})-(\mathrm{H})] 487 \mathrm{~nm}$. Samples (A) and (E) parent USY/Tb ${ }^{3+}$, (B) and (F) USY $/ \mathrm{Tb}^{3+} /$ vinyl, (C) and (G) USY $/ \mathrm{Tb}^{3+} /$ phenyl, and (D) and (H) USY $/ \mathrm{Tb}^{3+} / \mathrm{C}_{16}$.

silylation with vinyl and phenyl groups on terbium PL decays were negligible, e.g., their PL decays were similar to that corresponding to the parent zeolite. Therefore, for comparison, Fig. 2 illustrates only the PL decays of terbium in parent (USY $/ \mathrm{Tb}^{3+}$ ) and USY $/ \mathrm{Tb}^{3+} / \mathrm{C}_{16}$. From the data gathered in Table I, it results that for vinyl and phenyl silylating agents, the surface area parallels the loading of these compounds onto the zeolite surface, while for $\mathrm{C}_{16}$ silylating agent, although the number of docked molecules is smaller, the size of the organic ligand is covering more efficiently the zeolite surface. Further, silylation with $\mathrm{C}_{16}$ group zeolites caused a change in the relative ratios of the FT-IR bands within $3700-3100 \mathrm{~cm}^{-1}$ range indicating that the reaction presented in Scheme 1 occurs partially with the-OH groups attached to the terbium species.

The measured terbium PL lifetime $\tau$ related to the emission from the metastable ${ }^{5} D_{4}$ state is generally described as

$$
\tau=1 /\left(k_{\text {rad }}+k_{\text {nonrad }}+k_{\text {norad }}\right),
$$

where $k_{\text {rad }}$ is the natural radiative rate constant, $k_{\text {nonrad }}$ is the rate constant for the nonradiative relaxation processes due to the $-\mathrm{OH}$ coupling, and $k_{\text {nonrad }}$ is the rate constant for the radiationless relaxation processes due to other processes than the $-\mathrm{OH}$ coupling. $k_{\text {nonrad }}$ is proportional to the number of $\mathrm{OH}$ groups contained in the inner coordination shell of the terbium ion ${ }^{27}$ while $k_{\text {nonrad }}$ may include resonant ion-ion interactions located in close proximity or/and nonradiative energy transfer to host defects/impurities. Zeolite framework exhibits only low energy phonons, ${ }^{8}$ which are not expected to significantly contribute to the nonradiative deactivation of $\mathrm{Tb}^{3+}$ PL. Further, quenching of ${ }^{5} D_{4}$ related emission due to ion-ion interactions can be excluded given the low $\mathrm{Tb}^{3+}$ concentration and lack of energy resonances involving this metastable level. ${ }^{28}$ We therefore attribute the nonexponential shape of terbium PL decays to the existence of multiple terbium species. The longer PL decays measured with $\mathrm{USY} / \mathrm{Tb}^{3+} / \mathrm{C}_{16}$ relative to those corresponding to the parent or USY/ $\mathrm{Tb}^{3+} /$ vinyl and USY $/ \mathrm{Tb}^{3+} /$ phenyl zeolites may be explained by the increased protection of terbium ions against water molecules [i.e., reduction of the $k_{\text {nonrad" }}$ in Eq. (3)].

\section{Photoluminescence spectra}

First, TRES were analyzed paying attention to changes in spectral shape, presence of spectral shift, or appearance of the new peaks with time, which are generally used as an indicative of the system heterogeneity. ${ }^{29}$ The existence of a heterogeneous terbium distribution was readily ascertained in the TRES behavior regardless USY zeolite or the excitation wavelength. However, to specify the number of terbium species responsible for the observed TRES behavior, is not straightforward. Figure 3 illustrates the time evolution of the TRANES for USY/terbium zeolites. Compared to TRES, (peak normalized) TRANES has a physical significance. An isoemissive point in TRANES occurs at the wavelength $\left(\lambda_{\text {iso }}\right)$ where the ratio of the wavelength-dependent radiative rates $\left[k_{r 1}\left(\lambda_{\text {iso }}\right) / k_{r 2}\left(\lambda_{\text {iso }}\right)\right]$ is equal to the ratio of the total radiative rates $\left(k_{r 1} / k_{r 2}\right)$ of the emitting species ${ }^{5,6,13}$

$$
\frac{k_{r 1}\left(\lambda_{\text {iso }}\right)}{k_{r 2}\left(\lambda_{\text {iso }}\right)}=\frac{k_{r 1}}{k_{r 2}} \text {. }
$$

An isoemissive point in TRANES thus indicates that the observed PL emission from the sample is due to two species only, irrespective of the origin of the two species or the excited-state dynamics. There are two main features of interest which should be noticed from Fig. 3: (i) the presence of one isoemmisive point located at $547 \mathrm{~nm}$ regardless USY zeolite or excitation wavelength which strongly advocates for the existence of two main terbium species and (ii) the presence of a fast decaying species with emission centered at approximately $544 \mathrm{~nm}$.

To further check on the number of terbium species, we also applied SVD to the TRES data, (Fig. 4, exemplified for the parent USY/terbium). Five significant singular values 

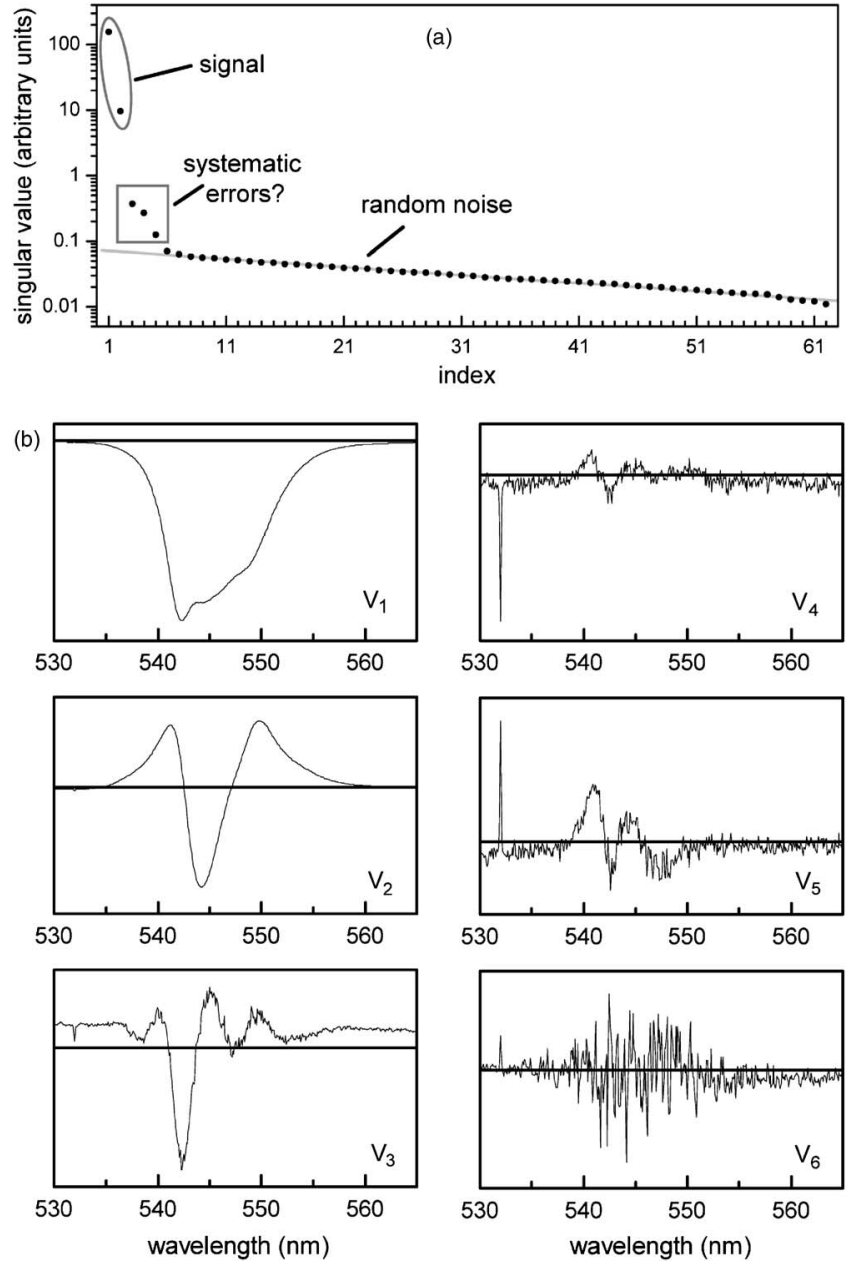

FIG. 4. TRES SVD analysis corresponding to the parent $\mathrm{USY} / \mathrm{Tb}^{3+}$ zeolite excited at $487 \mathrm{~nm}$. (a) Singular values as a function of the decomposition number index. Index refers to the number of signal components. (b) Abstract orthonormal spectra corresponding to the first six singular values.

above the noise level are observed from Fig. 4(a), which in principle can be attributed to five spectroscopic species. The contribution of the random noise component was easily identified by the exponential decrease with the index number or signal components [gray line in the Fig. 4(a)] and shape of its corresponding abstract spectra [see for instance, Fig. 4(b), $\left.V_{6}\right]$. The five significant singular values can be originated either from real species or by nonrandom systematic errors in the data. From the five significant components, only the first two were undoubtedly assignable to signal components, based on their high singular values [see Fig. 4(a)] but mainly from the shape of their corresponding abstract spectra [see Fig. 4(b), $V_{1}$ and $\left.V_{2}\right]$. On the other hand, the fourth and fifth components were clearly assigned from their associated abstract spectra to systematic errors in the form of data spikes [see Fig. 4(b), $V_{4}$ and $V_{5}$ ]. However, some of its features (e.g., a negative band at $542 \mathrm{~nm}$ ) could correspond either to systematic errors (e.g., detection nonlinearities) or to a true signal. In the latter case, we should conclude that three signal singular values and hence three luminescent species are contributing to the data. However, since the value of the third singular value is much smaller than the first two, two of the species could have a similar spectrum. Generally, similar results were obtained regardless zeolite or the excitation wavelength.

\section{Photoluminescence decays}

As the number of the discrete exponentials needed for a proper fitting of the terbium PL decays in USY zeolites was between three and four, such an approach was discarded. We used instead the MaxEnt, which offers the advantage of handling complex multiexponential or nonexponential decays without a priori assumption of the shape of the lifetime distribution. To identify the noise-induced features, we performed Monte Carlo simulations, which provided the expected variance in the maximum entropy distributions caused by the presence of noise. ${ }^{21}$ Three decays with positive amplitude were reproducible for all USY zeolites at 337, 390, and $487 \mathrm{~nm}$ excitation wavelengths. For an excitation wavelength of $487 \mathrm{~nm}$, the time constants ranged between 410 and $440 \mu \mathrm{s}, 1000$ and $1100 \mu \mathrm{s}$, and 1.9 and $2.1 \mathrm{~ms}$ for the parent and silylated zeolites, respectively. The corresponding amplitudes were $0.35 / 0.57 / 0.08$ for the parent, $\mathrm{USY} / \mathrm{Tb}^{3+} /$ vinyl and USY $/ \mathrm{Tb}^{3+} /$ phenyl zeolites and $0.25 /$ $0.66 / 0.09$ for the USY $/ \mathrm{Tb}^{3+} / C_{16}$. Two points of interest are evidenced form the MaxEnt results: (i) the predominant contribution to the total PL is given by the fast $(410-440 \mu \mathrm{s})$ and medium decay components $(1000-1100 \mu s)$ and (ii) following silylation, the amplitude of the medium decay component increased mainly at the expense of that of the fast decay component (e.g., it increased from 0.57 (parent USY/ terbium) to 0.66 (USY/terbium/ $\mathrm{C}_{16}$ ). Silylation was considered as a method to protect USY zeolite surface against humidity and hence to preserve the PL properties of the exchanged terbium species more or less at the values obtained after the calcination. The average PL lifetime [calculated with Eq. (2)] of terbium in silylated USY $/ \mathrm{Tb}^{3+} / \mathrm{C}_{16}$ is approximately $1020 \mu \mathrm{s}$ while for the parent and $\mathrm{USY} / \mathrm{Tb}^{3+} /$ vinyl and $\mathrm{USY} / \mathrm{Tb}^{3+} /$ phenyl zeolites the terbium PL lifetime was reduced by $15 \%$ at $865 \mu \mathrm{s}$.

Starting from the number, time constants, and bandwidth of the luminescent related components provided by MaxEnt, the corresponding least-squares DAS were obtained (Fig. 5). DAS have also a physical significance as they represent the spectral distributions of the individual terbium species which contribute to the total PL, which are uniquely linked to the decay components. To emphasize the difference in DAS derived for each decay component, the spectra were normalized and displayed in the Fig. 6 . The differences between the spectral shapes and full width at half maximum (FWHM) values of the fast decay $(410-440 \mu \mathrm{s})$ on one side, and medium (1000-1100 $\mu \mathrm{s})$, and slow (1.9-2.1 ms) decay components on the other side, clearly indicate that there are at least two main terbium species of a different origin. The amplitude spectra shape and time constants for the decay components were relatively similar regardless sample or excitation wavelength, differing mostly in their intensity. The same analysis was performed by globally fitting the data above $100 \mu$ s to three decay components (GExpNLLS method), providing the least-square time constants and DAS. 

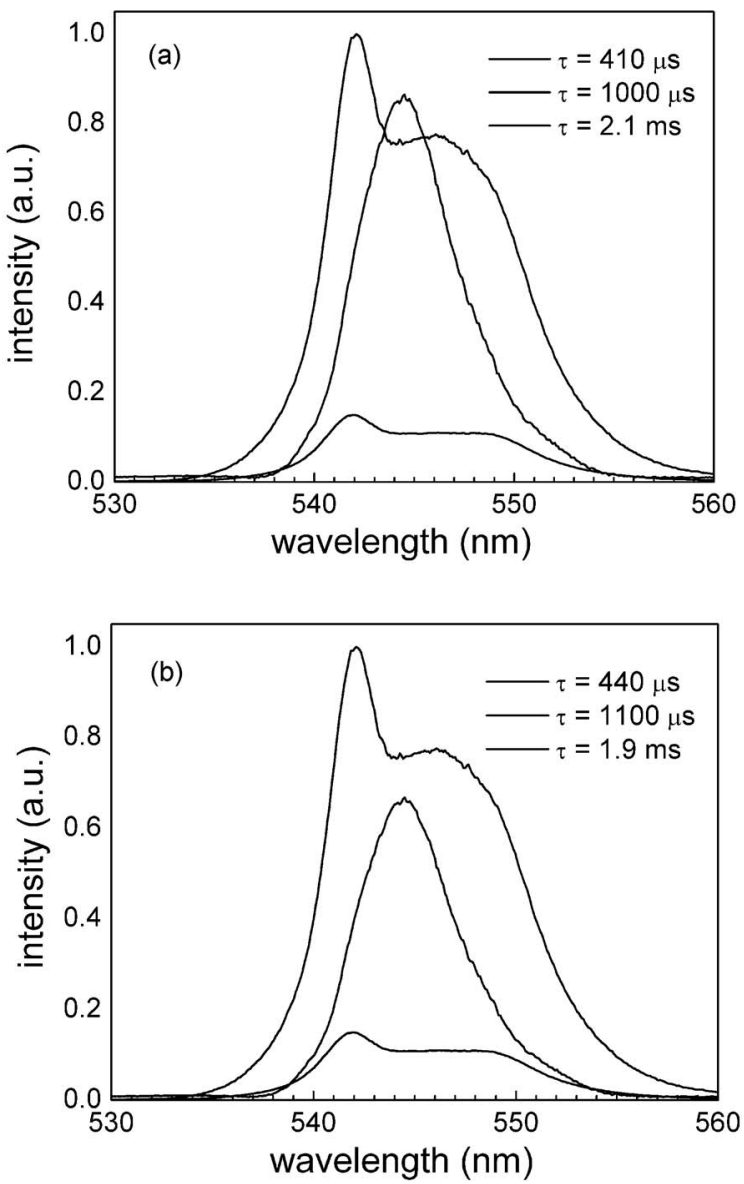

FIG. 5. DAS corresponding to TRES spectra of parent USY/terbium (a) and USY/terbium/ $\mathrm{C}_{16}$ (b) zeolites following excitation at $487 \mathrm{~nm}$.

In most cases, the obtained results were similar to those obtained by MaxEnt, although GExpNLLS clearly failed for the vinyl data sets, providing unrealistic time constants and/or DAS (not shown). As it is generally accepted, the exponential analysis by MaxEnt is more reliable in nonideal conditions, introducing an enhanced robustness in the analysis in comparison to GExpNLLS. Further improvements in data analysis of PL data will consider the substitution of the least-squares methods by more robust ones, such as leastabsolute methods ${ }^{30}$ and the pre-processing step removing outliers from the data.

Although there is a number of studies devoted on the lanthanides-exchanged $\mathrm{Y}$ zeolites, the exact location of the ions in zeolite framework still needs further clarification. It is acknowledged that the lanthanide ions are initially introduced in the large $1.3 \mathrm{~nm}$ diameter cages (supercages) where the ion is fully hydrated and not coordinated by the framework. ${ }^{31-34}$ Subsequent thermal treatment at temperatures as low as $80{ }^{\circ} \mathrm{C}$ initiates a process of dehydration/ dehydroxylation of the cation, which allows it to migrate through the six-ring openings of the smaller cages (or sodalites) and reside in the SI' sites in sodalite cages. ${ }^{35-46}$ The presence of a fraction of terbium ions in the supercages of $\mathrm{Y}$ zeolite even after calcination at $450{ }^{\circ} \mathrm{C}$ was also speculated according to Ref. 34 . Other studies claim that lanthanides are found only in sodalites sites as a result of a completed migration process. ${ }^{40}$

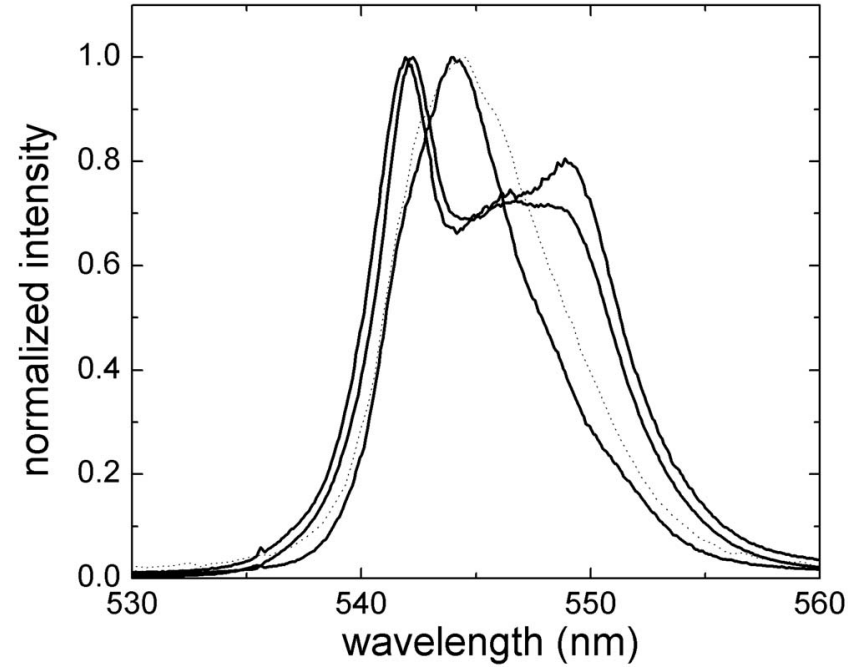

FIG. 6. Comparison between the DAS related to the fast, medium and long decaying terbium species in USY $/ \mathrm{Tb}^{3}$ (see also Fig. 5). Also represented is the experimental PL spectrum corresponding to the hydrated USY/ $\mathrm{Tb}^{3+}$ zeolites (dotted line).

Following heating at $450 \mathrm{C}^{\circ}$, we found that the terbium PL intensity increased and the decay was slowed. Further, a detectable structuring of the $545 \mathrm{~nm}$ based transition was observed together with the increasing of the corresponding FWHM value from $\sim 6$ to $\sim 10 \mathrm{~nm}$. A combination of the crystal-field modification and the $-\mathrm{OH}$ groups removal from the first coordination shell of terbium is most appropriate to explain these PL changes after the thermal treatment. Such changes are generally evidenced in the literature for all the lanthanide's exchanged zeolites following thermal treatments. For the case of zeolite Y, because the diameter of a hydrated $\mathrm{Ln}^{3+}$ cation is $7.9 \AA$ (Ref. 41) and the diameter of the six-ring window to the sodalite cage is only $2.5 \AA$ the partial loss of their hydration shell should occur during the migration of lanthanum cations into the sodalite cage.

Albeit PL spectroscopy cannot offer unambiguous data on the exact location of terbium ions within $\mathrm{Y}$ zeolite framework or the extent of the migration process, we try to evidence that a combination of the analysis methods such those presented here can determine the terbium distribution (e.g., number of species) and the local environment at the dopant sites described in terms of characteristic PL spectra and decays. It is clear from the decay-associated spectra represented in Fig. 6 that while the amplitude spectra of the medium and slow decay show a similar dip structure (at $\sim 543 \mathrm{~nm})$ and FWHM values $(\sim 10-11 \mathrm{~nm})$, the faster decay shows a much narrower amplitude spectrum (FWHM at $\sim 6 \mathrm{~nm}$ ) with a single maximum at $544 \mathrm{~nm}$. The corresponding decay-associated spectrum of the fast decaying species has similar spectral shape and FWHM value with the experimental PL spectrum measured with the hydrated (asexchanged) USY/terbium, also included in Fig. 6. Indeed, both emission peaks are at $\sim 544 \mathrm{~nm}$, a similar value within $0.5 \mathrm{~nm}$ to that of the fast decaying emission as seen in TRANES behavior (Fig. 3). To further check on the nature of the fast decaying species, we have subtracted from the experimental PL spectrum of terbium-USY measured at $5 \mu \mathrm{s}$ 
after the laser pulse (prompt spectrum) the delayed PL spectrum measured at $2500 \mu \mathrm{s}$ after the laser pulse. The resulted difference spectrum centered at $545 \mathrm{~nm}$ has similar shape, FWHM, and peak values to those measured with the hydrated terbium-USY or the DAS corresponding to the fastdecaying species. We have also measured the PL decay of the hydrated USY/terbium (represented in Fig. 2) and fitted with a biexponential function. The results indicated that most terbium ions $(\sim 90 \%)$ have PL lifetimes at about $390 \mu \mathrm{s}$, i.e., terbium ions can be considered as fully hydrated. This value comes close to the lifetime's values associated with the fast decaying terbium species $(410-440 \mu \mathrm{s})$. In consequence, all the data obtained with experimental timeresolved PL spectra (Figs. 3 and 6) and decay as well as the MaxEnt lifetimes analysis and DAS (Fig. 5) converged to the existence of a short-lifetime terbium species. Since in Y zeolites water molecules were found at sites II' (sites which are also located in the sodalite cage but close to the supercage) even for samples calcined above $300{ }^{\circ} \mathrm{C}$ (Ref. 42) the fast decaying species may be ascribed to the terbium ions located in these sites. Note that no precaution was considered to protect USY samples against atmosphere moist following calcination or silylation. The medium decay component at $1000-1100 \mu \mathrm{s}$ is associated to the terbium ions located in zeolite pores with coordination to both water molecule and framework oxygens. Its dip structure as seen from the associated decay spectra or the experimental time-resolved PL spectrum at long time delays (Figs. 5 and 6) is attributed to changes in the crystal field at the terbium sites following calcination and the partial loss of the water ligands. According to the literature consensus, the location of this species within zeolite framework can be associated to SI' sites in the sodalite cages. ${ }^{35-46}$

The existence of two main terbium species is supported by the presence of an isoemmisive point in the TRANES of all USY/terbium zeolites at 337 and $487 \mathrm{~nm}$ excitation wavelengths (Fig. 3) and partially by SVD (Fig. 4). A third species, probably constituted as nano- $\mathrm{Tb}_{2} \mathrm{O}_{3}$-type structures on the USY surface, may be associated to the longest decay component at $1.9-2.1 \mathrm{~ms}$. No experimental evidence for the third, long lifetime terbium species could be however inferred from the PXRD or DR-UV-visible spectra.

A consistent comparison between our values of the PL lifetimes of terbium-exchanged $\mathrm{Y}$ zeolites and those reported in literature is hindered by the different synthesis history and treatment conditions imposed to samples prior to the PL measurements. Further, to the best of our knowledge, there is no report in the literature on the time-resolved PL properties of the terbium-exchanged USY zeolites. For the terbiumexchanged Y zeolites, following laser excitation at $490 \mathrm{~nm}$ a mono exponential decay with PL lifetime value of $2.4 \mathrm{~ms}$ was measured. ${ }^{39}$ Here, the sample was calcined at $450{ }^{\circ} \mathrm{C}$ for $8 \mathrm{~h}$. and measured at room temperature. Serra et al., have reported a value of $570 \mu$ s for the PL lifetime of a terbiumexchanged Y zeolite. In this work, the sample was calcined at $200{ }^{\circ} \mathrm{C}$ for $2 \mathrm{~h}$ and the PL was measured at $77 \mathrm{~K}^{23}$

Future work will extend this approach to the analysis of time-resolved PL data obtained with terbium or europium ions in various sol-gel microporous-mesoporous materials.

\section{CONCLUSION}

A detailed analysis of the time-resolved PL spectra of parent or nonsilylated and silylated terbium-USY zeolites was pursued. Except for a questionable low amplitude, long lifetime species with lifetime of 1.9-2.1 ms, we have demonstrated that two main terbium species coexist in all parent and silylated USY zeolites, with lifetimes of 410-440 and $1000-1100 \mu \mathrm{s}$, respectively. The number and their distinct PL properties were strongly supported by the experimental time-resolved PL spectra as well as analysis results based on the single-value decomposition, maximum-entropy and the decay associated spectra.

\section{ACKNOWLEDGMENTS}

The authors are grateful to B. Gagea from K. U. Leuven, Belgium, for the samples synthesis.

${ }^{1}$ T. Justel, D. U. Wiechert, C. Lau, D. Sendor, and U. Kynast, Adv. Funct. Mater. 11, 105 (2001)

${ }^{2}$ C. Sanchez, B. Lebeau, F. Chaput, and J. P. Boilot, Adv. Mater. (Weinheim, Ger.) 15, 1969 (2003).

${ }^{3}$ Y. Wang, H. Li, W. Zhang, and B. Liu, Mater. Lett. 62, 3167 (2008).

${ }^{4}$ A. Monguzzi, G. MacChi, F. Meinardi, R. Tubino, M. Burger, and G. Calzaferri, Appl. Phys. Lett. 92, 123301 (2008).

${ }^{5}$ C. Tiseanu, M. U. Kumke, V. I. Parvulescu, A. Gessner, B. Gagea, and J. A. Martens, J. Phys. Chem. B 110, 25707 (2006).

${ }^{6}$ C. Tiseanu, M. U. Kumke, V. I. Parvulescu, A. S. R. Koti, B. Gagea, and J. A. Martens, J. Photochem. Photobiol., A 187, 299 (2007).

${ }^{7}$ C. Tiseanu, B. Gagea, V. I. Parvulescu, V. Lórenz-Fonfría, A. Gessner, and M. U. Kumke, Langmuir 23, 6781 (2007)

${ }^{8}$ H. Maas, A. Currao, and G. Calzaferri, Angew. Chem., Int. Ed. 41, 2495 (2002).

${ }^{9}$ R. Singh and P. K. Dutta, Microporous Mesoporous Mater. 32, 29 (1999).

${ }^{10}$ W. G. Song, G. H. Li, V. H. Grassain, and S. C. Larsen, Environ. Sci. Technol. 39, 1214 (2005).

${ }^{11}$ N. K. Jeong, H. S. Kim, and K. B. Yoon, Langmuir 21, 6038 (2005).

${ }^{12}$ R. W. Hendler and R. I. Shrager, J. Biochem. Biophys. Methods 28, 1 (1994).

${ }^{13}$ A. S. R. Koti and N. Periasamy, J. Chem. Phys. 115, 7094 (2001).

${ }^{14}$ K. H. Müller and T. Plesser, Eur. Biophys. J. 19, 231 (1991).

${ }^{15}$ J. M. Beechem, Methods Enzymol. 210, 37 (1992).

${ }^{16}$ J. R. Knutson, J. M. Beechem, and L. Brand, Chem. Phys. Lett. 102, 501 (1983).

${ }^{17}$ I. H. van Stokkum, D. S. Larsen, and R. van Grondelle, Biochim. Biophys. Acta 1657, 82 (2004).

${ }^{18}$ A. K. Livesey, P. Licinio, and M. Delaye, J. Chem. Phys. 84, 5102 (1986).

${ }^{19}$ A. K. Livesey and J. C. Bronchon, Biophys. J. 52, 693 (1987).

${ }^{20}$ V. A. Lórenz-Fonfría and H. Kandori, Appl. Spectrosc. 61, 428 (2007).

${ }^{21}$ V. A. Lórenz-Fonfría and H. Kandori, Appl. Spectrosc. 61, 74 (2007).

${ }^{22}$ P. C. Hansen, SIAM J. Sci. Comput. (USA) 14, 1487 (1993).

${ }^{23}$ O. A. Serra, E. J. Nassar, G. Zapparolli, and I. L. Rosa, J. Alloys Compd. 225, 63 (1995).

${ }^{24}$ W. H. Press, S. A. Teukolsky, W. T. Vetterling, and P. Flannery, Numerical Recipes in C: The art of Scientific Computing, 2nd ed. (Cambridge University Press, New York, 1992).

${ }^{25}$ H. G. Karge, Verified Synthesis of Zeolitic Materials, 2nd ed., edited by H. Robson (Elsevier, New York, 2001).

${ }^{26}$ G. Wakefield, H. A. Keron, P. J. Dobson, and J. L. Hutchison, J. Phys. Chem. Solids 60, 503 (1999).

${ }^{27}$ W. D. Horrocks, Jr. and D. R. Sudnick, J. Am. Chem. Soc. 101, 334 (1979).

${ }^{28}$ C. Armellini, M. Ferrari, M. Montagna, G. Pucker, C. Bernard, and A. Monteil, J. Non-Cryst. Solids 245, 115 (1999).

${ }^{29}$ J. R. Lakowicz, Principles of Fluorescence Spectrometry (Plenum, New York, 1983).

${ }^{30}$ A. Tarantola, Inverse Problem Theory, Society for Industrial and Applied Mathematics (Society for Industrial and Applied Mathematics, Philadelphia, PA, 2005).

${ }^{31}$ J. M. Bennett and J. V. Smith, Mater. Res. Bull. 4, 343 (1969). 
${ }^{32}$ D. H. Olson, G. T. Kokotailo, and J. F. Charnell, J. Colloid Interface Sci. 28, 305 (1968).

${ }^{33}$ S. L. Suib, R. P. Zerger, G. D. Stucky, T. I. Morrison, and G. K. Shenoy, J. Chem. Phys. 80, 2203 (1984).

${ }^{34}$ M. Alvaro, V. Fornes, S. Garcia, H. Garcia, and J. C. Scaiano, J. Phys. Chem. B 102, 8744 (1998).

${ }^{35}$ E. F. T. Lee and L. V. C. Rees, Zeolites 7, 143 (1987).

${ }^{36}$ E. F. T. Lee and L. V. C. Rees, Zeolites 7, 545 (1987).

${ }^{37}$ D. Keir, E. F. T. Lee, and L. V. C. Rees, Zeolites 8, 228 (1988).

${ }^{38}$ M. D. Baker, M. O. Olken, and G. A. Ozin, J. Am. Chem. Soc. 110, 5709 (1988).

${ }^{39}$ S. Hashimoto, S. Kirikae, and S. Tobita, Phys. Chem. Chem. Phys. 4, 5856 (2002).
${ }^{40}$ H. Klein, H. Fuess, and M. J. Hunger, J. Chem. Soc., Faraday Trans. 91, 1813 (1995).

${ }^{41}$ C. F. Lin and K. J. Chao, J. Phys. Chem. 95, 9411 (1991).

${ }^{42}$ P. Norby, F. I. Poshni, A. F. Gualtieri, J. C. Hanson, and C. P. Grey, J. Phys. Chem. B 102, 839 (1998).

${ }^{43}$ J. M. Bennett, J. V. Smith, and C. L. Angell, Mater. Res. Bull. 4, 77 (1969).

${ }^{44}$ A. K. Cheetham, M. M. Eddy, and J. M. Thomas, J. Chem. Soc., Chem. Commun. 1984, 1337.

${ }^{45}$ K. Gaare and D. Akporiaye, J. Phys. Chem. B 101, 48 (1997).

${ }^{46}$ D. Brennan, R. G. Bell, C. R. A. Catlow, and R. A. Jackson, Zeolites 14, 650 (1994) 